\section{Repeated Dientamoeba fragilis infections: a case report of two families from Sydney, Australia}

Damien Stark, ${ }^{1,2}$ Joel Barratt,, John Ellis, ${ }^{1}$ John Harkness, ${ }^{1,2}$ Deborah Marriott ${ }^{1,2}$

'Division of Microbiology, SydPath, St. Vincent's Hospital, Sydney, Australia; ${ }^{2}$ Department of Medical and Molecular Biosciences, University of Technology Sydney, Broadway, Australia

\section{Abstract}

We report cases of two unrelated families who both presented with recurrent Dientamoeba fragilis infections. Subsequent antimicrobial therapy resulted in the clearance of $D$. fragilis and total resolution of gastrointestinal symptoms in both families. This report highlights the potentially recurrent nature of $D$. fragilis infections and the need for laboratories to routinely test for this organism.

\section{Introduction}

Dientamoeba fragilis is a pathogenic amoeboid protozoan parasite that is closely related to the Trichomonads. The parasite has been shown to cause gastrointestinal disease in a wide range of patient groups, has a world-wide distribution and is often more prevalent than Giardia. ${ }^{1,2}$ Chronic symptoms have been reported previously in patients presenting with $D$. fragilis infection. ${ }^{3}$ One study found that $32 \%$ of $D$. fragilis infected patients had persistent diarrhoea and associated symptoms of greater than 2 weeks duration. ${ }^{4}$ We report two separate cases of repeated $D$. fragilis infection in family members sharing the same residence who by genotyping were determined to be infected by different strains of $D$. fragilis.
Parasitology testing was performed on faecal samples fixed in Sodium acetate acetic acid formalin and permanently stained using a modified iron-haematoxylin stain as previously described. ${ }^{4}$ Diagnosis of $D$. fragilis infection was made based on the finding of binucleate, pleomorphic, granular, amoeboid cells, typical of $D$. fragilis in the initial stained smear. ${ }^{4}$ The patient was treated with metronidazole, symptoms improved, and on follow up examination of stool sample $(n=1)$ no parasites were detected, indicating that the infection was successfully cleared. Several months after the initial presentation and subsequent successful treatment of the $D$. fragilis infection the patient presented again with gastrointestinal complaints including a variation in bowel motions from watery diarrhoea to unformed faecal motions. Stool samples were resubmitted again and underwent routine bacteriological culture, and permanent staining of fixed faecal smears for the identification of protozoan parasites. In addition a portion of stool sample underwent DNA extraction and PCR using specific primers targeting the SSUrDNA of $D$. fragilis as previously described. ${ }^{5}$ No bacterial pathogens were identified, while microscopic analysis of the stained smears detected $D$. fragilis trophozoites and the $D$. fragilis $\mathrm{PCR}$ was also positive for $D$. fragilis DNA. After the diagnosis of dientamoebiasis was made the patient was treated with doxycycline and iodoquinol. The patients symptoms resolved after treatment and subsequent stool samples submitted post treatment $(\mathrm{n}=2)$ were negative for Dientamoeba by both microscopy and PCR.

At the same time the patient's mother, a 75year-old female (Patient 2), who cohabited at the same residence as her son also presented with a history of gastrointestinal symptoms including bouts of diarrhoea and unformed stools, faecal urgency, digestive problems and food intolerance. Microbiological analysis was undertaken on stool specimens including microbial cultures, parasitology testing, and $D$. fragilis specific PCR. Bacterial cultures were negative and the permanent stained faecal
Correspondence: Damien Stark, Department of Microbiology, St.Vincent's Hospital, Darlinghurst 2010, NSW, Australia. E-mail: dstark@stvincents.com.au

Key words: Dientamoeba fragilis.

Contribution: DS, main author, molecular analysis, microbiological and laboratory testing; JB, molecular analysis, microbiological and laboratory testing testing article input, proof reading; JE, molecular analysis, article input, proof reading; $\mathrm{JH}$, clinical input, patient follow up article input, proof reading; DM, clinical input and treatment of patients, article input, proof reading.

Received for publication: 7 September 2009.

Revision received: 26 October 2009.

Accepted for publication: 28 0ctober 2009.

This work is licensed under a Creative Commons Attribution 3.0 License (by-nc 3.0).

(C) Copyright D. Stark et al., 2009

Infectious Disease Reports 2009; 1:e4

doi:10.4081/idr.2009.e4

smears of the mother's stool specimen demonstrated the presence of Blastocystis hominis. No Dientamoeba trophozoites were detected by microscopy. However a positive $D$. fragilis PCR result was obtained from the mother's stool demonstrating the presence of Dientamoeba DNA. Sequencing of the PCR products from mother and son was undertaken as previously described. ${ }^{5}$ The Dientamoeba-specific PCR product obtained from the stool of the patient's mother was sequenced and found to have identical SSU rDNA sequence as that obtained from her son. ${ }^{5}$ Given that the SSU rRNA gene displays insufficient variability to be used as a definitive epidemiological marker the previously described $D$. fragilis typing method of Cprofiling was undertaken on both samples. ${ }^{6}$ Both $D$. fragilis samples yielded different Cprofiles indicating the strains were not genotypically identical (Figure 1). Patient 2 was

\section{Case Report}

\section{Case 1}

A 41-year-old male (Patient 1) presented with a history of chronic diarrhoea and weight loss over a period of several months. A faecal sample was collected and routine bacteriological cultures were performed along with investigation for parasites. Due to the chronic nature of the condition no virology was performed on the samples given the acute, selflimiting nature of viral gastroenteritis. No bacterial pathogens were detected by culture.

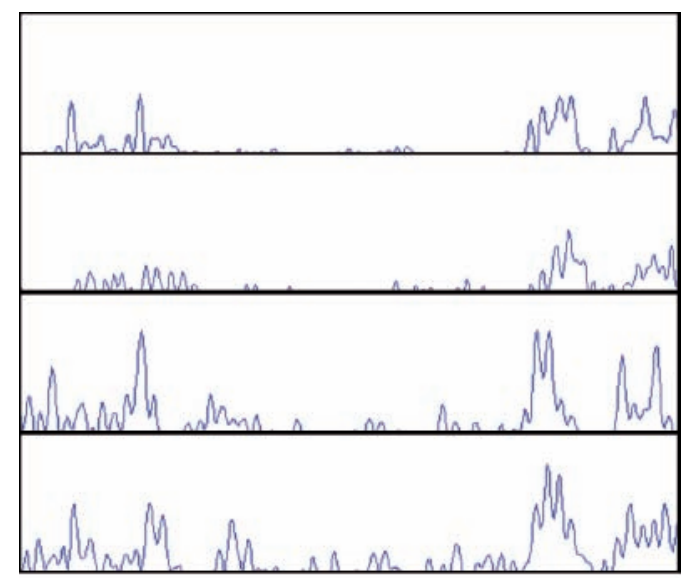

Figure 1. C-profiles from Case 1 and Case 2. From top to bottom Case 1 patient 1, patient 2, Case 2 father, Case 2 son. 
treated with secnidazole, nitazoxanide and doxycycline, which resulted in total parasitological clearance and resolution of the patient's symptoms. Follow-up samples collected after treatment and again some 3 months later failed to detect any $D$. fragilis either by microscopy or by molecular methods. As Enterobius vermicularis has been proposed by some as a possible vector for transmission of $D$. fragilis, multiple sticky-tape tests were collected from both $D$. fragilis infected patients. A total of 6 tape tests were collected and examined; no $E$. vermicularis ova were detected.

\section{Case 2}

A family comprising of the father (58 years of age), mother (49 years of age), son (13 years of age) and daughter (10 years of age) presented to their local general practitioner with gastrointestinal complaints after a holiday to Tasmania. The gastrointestinal symptoms included diarrhoea, abdominal pain and bloating. One month after the holiday in April 2008, all four patients submitted stool samples for routine bacteriological cultures along with investigation for parasites. No bacterial pathogens were isolated. However $D$. fragilis was detected by microscopy of permanently stained faecal smears as previously described, in three out of the four patients (father and both children). Both children also presented with a peripheral eosinophilia. In May all four patients were treated with metronidazole and symptoms resolved. No follow-up stool samples were collected to check for clearance of parasite. Four months later in September, three of the family (father, son and daughter), presented with gastrointestinal symptoms, faecal samples were resubmitted and $D$. fragilis was detected by permanent stained microscopy in all three patients with the father also having the non-pathogenic flagellate Chilomastix mesnili present. All three patients were subsequently treated with paramomycin for 10 days. Follow-up stool samples were collected one month after cessation of treatment and no $D$. fragilis was detected by microscopy. Five months later the family once again presented with gastrointestinal complaints and microbiological analysis of faecal samples were performed. Dientamoeba fragilis was detected in 2/4 family members (father and son) by microscopy of permanent stained smears,. Faecal samples were then collected from all members of the family and an RT-PCR was performed as described. ${ }^{7}$ The PCR assay detected $D$. fragilis in all family members, including the two members who were microscopy negative for $D$. fragilis only 5 days earlier. In order to fingerprint the $D$. fragilis strains, C-profiling was used to determine if the infection was from a single source. Only two samples, from the father and son were able to be fingerprinted using this technique and both strains were shown to be genotypically different (Figure 1). Treatment was initiated for all family members (paramomycin for 10 days). All family members reported resolution of symptoms and follow-up molecular analysis of stool samples one month later detected no $D$. fragilis DNA. Follow-up two months later showed that the family was still symptom and parasite free.

\section{Discussion}

Dientamoeba fragilis is a protozoan parasite that has recently emerged as an important cause of parasitic gastrointestinal disease.,4 Recent studies have shown the organism to be widespread with relatively high prevalence rates ranging from $8.9 \%$ to $16.8 \%$ in developed regions of the world ${ }^{8-10}$ Gastrointestinal symptoms attributed to Dientamoeba infection most commonly include diarrhoea and abdominal pain, with chronic infection often reported. ${ }^{4,11,12}$ Numerous studies have shown antimicrobial therapy targeting and eliminating $D$. fragilis will result in marked clinical improvement for patients suffering from dientamoebiasis. ${ }^{3,12,13}$

All patients from both families presented with a repeated $D$. fragilis infection over prolonged periods of time. Bacterial etiological agents were excluded by routine testing. Dientamoeba was detected by either microscopy or PCR (or both) in the patients' samples. The PCR detected $D$. fragilis infections that would have been subsequently missed if microscopy only had been used. Molecular testing has been shown to provide excellent sensitivity and specificity when compared to microscopy for the detection of $D$. fragilis and provides an additional diagnostic tool for laboratories with this capability. The symptoms described in all patients included a variation in bowel motions with bouts of diarrhoea, unformed stool samples and faecal urgency along with stomach pain and cramps.

In case 1 , patient 1 had a previous $D$. fragilis infection which initially seemed to respond to therapy; however the patient presented again several months later. Whether the latter infection was a new infection from a different source, a reinfection from the same source or even treatment failure is unknown. Samples from the first episode of infection were not collected for molecular analysis. As it was possible that the reinfection was due to selection of resistant $D$. fragilis strains and subsequent treatment failure both patients were treated with combination therapy to eradicate the organism. One patient was treated with doxycline and iodoquinol while the other secnidazole, nitazoxanide and doxycycline. Both patients responded to treatment with eradication of the organism and resolution of symptoms. The patient who was treated with sec- nidazole, nitazoxanide and doxycycline did complain of side effects from the antimicrobial agents.

The patients from case 2 had gastrointestinal complaints for over a year and even though treatment was given on several occasions to family members, $D$. fragilis and gastrointestinal complaints returned. As some members of the family had resolution of symptoms and clearance of $D$. fragilis from stools after treatment it must be assumed that the patients were getting re-infected. After treatment with metronidazole after the initial $D$. fragilis "outbreak" amongst the family, no follow-up stool samples were examined for clearance of the parasite. Subsequent symptomatic presentations occurred only in the father and children. When all family members were treated at the same time, the symptoms and parasites were cleared, and to-date, several months later, the family remains symptom and parasite free.

Molecular testing, using C-profiling, was performed and both $D$. fragilis isolates from case 1 (patient 1 and 2) were shown to be different, indicating infection from a different source. C-profiling on case 2 samples also showed the strains were not identical. C-profiling is a method that has been used for the molecular epidemiological typing of $D$. fragilis that targets the internal transcribed spacer regions (ITS). ${ }^{6}$ The ITS regions have been used extensively for phylogenetic analyses and as a molecular epidemiological marker of other parasites and in particular members of the Trichomonadidae. ${ }^{6}$ The method detects intragenomic variation in the $D$. fragilis ITS region which leads from direct amplification of samples to sequencing fluorograms that are too complex to interpret, due to multiple ITS sequence variants in a single isolate. However, since the ITS regions of $D$. fragilis are extremely AT rich and the C-content is low by deleting fluorogram peaks representing the other nucleotides (A, T and G), it is possible to analyse $\mathrm{C}$ nucleotide residues producing chromatographs that are reproducible and easy to interpret. Bart et al. clearly demonstrated that the intragenomic variation of the ITS regions of $D$. fragilis can be used as a molecular epidemiological marker. ${ }^{6}$ In both cases the $D$. fragilis strains were shown to be genotypically different, so it must be assumed that both patients obtained the infection from a different source.

Sticky-tape tests collected from all patients were negative for $E$. vermicularis and so it must be assumed that infection occurred by direct transmission. Recent studies have not shown a role for $E$. vermicularis in transmission of $D$. fragilis. ${ }^{4}$

This report highlights the repeated nature of some $D$. fragilis infections. Given that the organism can be treated effectively with a number of antimicrobial agents, all laborato- 
ries should provide a parasitological service capable of detecting this organism. All family members or those living in the same residence should be screened for $D$. fragilis as asymptomatic carriers may provide an ongoing source of infection.

\section{References}

1. Vandenberg 0 , Peek R, Souayah H, et al. Clinical and microbiological features of dientamoebiasis in patients suspected of suffering from a parasitic gastrointestinal illness: a comparison of Dientamoeba fragilis and Giardia lamblia infections. Int J Infect Dis 2006;10:255-61.

2. Crotti D, D'Annibale ML, Fonzo G, et al. Dientamoeba fragilis is more prevalent than Giardia duodenalis in children and adults attending a day care centre in Central Italy. Parasite 2005;12:165-70.

3. Stark DJ, Beebe N, Marriott D, et al. Dientamoebiasis: clinical importance and recent advances. Trends Parasitol 2006;
22:92-6.

4. Stark D, Beebe N, Marriott D, et al. Prospective study of the prevalence, genotyping, and clinical relevance of Dientamoeba fragilis infections in an Australian population. J Clin Microbiol 2005;43:271823.

5. Stark D, Beebe N, Marriott D, et al. Detection of Dientamoeba fragilis in fresh stool specimens using PCR. Int J Parasitol 2005;35:57-62.

6. Bart A, van der Heijden HM, Greve S, et al. Intragenomic variation in the internal transcribed spacer 1 region of Dientamoeba fragilis as a molecular epidemiological marker. J Clin Microbiol 2008;46:3270-5.

7. Stark D, Beebe N, Marriott D, et al. Evaluation of three diagnostic methods, including real-time PCR, for detection of Dientamoeba fragilis in stool specimens. J Clin Microbiol 2006;44:232-5.

8. Stensvold CR, Arendrup MC, Molbak K, Nielsen HV. The prevalence of Dientamoeba fragilis in patients with suspected enteroparasitic disease in a metropolitan area in Denmark. Clin Microbiol Infect 2007;13:839-42.

9. Schuster H, Jackson BM. Prevalence of Dientamoeba fragilis amongst patients consulting complimentary medicine practitioners in the British Isles. J Clin Pathol 2008 0ct 24.

10. Rayan HZ, Ismail OA, El Gayar EK. Prevalence and clinical features of Dientamoeba fragilis infections in patients suspected to have intestinal parasitic infection. J Egypt Soc Parasitol 2007;37:599608.

11. Ito R, Sakagami J, Kataoka K, et al. Chronic diarrhea and protein-losing gastroenteropathy caused by Dientamoeba fragilis. J Gastroenterol 2004;39:1117-9.

12. Johnson EH, Windsor JJ, Clark CG. Emerging from obscurity: biological, clinical, and diagnostic aspects of Dientamoeba fragilis. Clin Microbiol Rev 2004; 17:553-70.

13. Vandenberg 0 , Souayah H, Mouchet F, et al. Treatment of Dientamoeba fragilis infection with paromomycin. Pediatr Infect Dis J 2007;26:88-90. 